\title{
The History of STDs
}

The series of papers which are to appear in Genitourinary Medicine over the next year or so and for which we have acted as editors, presents the history of sexually transmitted diseases in a variety of countries. We have tried to obtain papers on countries from a range of cultural regions: South, East and Southeast Asia, Southern Africa, North America, Western Europe and Latin America. The series offers readers both longitudinal or historical and cross-sectional or comparative perspectives on sexually transmitted diseases. The historical perspective, of course, permits us to see change over time, while the comparative viewpoint enables us, by highlighting differences in the social, economic, political and cultural context, to see more clearly the important role played by non-medical factors in shaping both disease patterns and attitudinal and policy responses to those patterns.

Contributors to the series have been requested to focus their chronological attention on the "modern" periodthe nineteenth and twentieth centuries, although some have chosen to outline in some detail historical developments in earlier eras. They have been asked to address at least three broad areas. They have been asked first to say something about the historical epidemiology of the classical venereal diseases, and, where possible, to provide information on historical trends in morbidity and mortality; second, to discuss the development of control policy, the evolution of therapy and the growth of preventive and curative facilities; and finally, to endeavour to explain how social attitudes and values have influenced both disease patterns and collective responses to them.

In its preoccupation with science, rationality and universal application, modern medicine tends to forget the cultural and social matrix in which communicable diseases are embedded. Arguably, this contextual aspect is particularly significant where the diseases are closely linked to human sexuality, surrounded as it is by powerful moral and emotional force fields. We believe that the series will inform and entertain but we hope that the historical and comparative approach may provide insights useful to the development of more effective controls of diseases which demonstrate considerable capacity to adapt and survive.

Senior Fellow, Department of Public Health

MILTON LEWIS University of Sydney, Australia

Consultant in Genitourinary Medicine

MICHAEL WAUGH Leeds, UK 